\title{
Results from the first one and a half years of the HAWC GRB program
}

\author{
Joshua Wood $^{* a}$ for the HAWC Collaboration ${ }^{b}$ \\ ${ }^{a}$ Department of Physics, University of Wisconsin, Madison, Wisconsin, USA \\ ${ }^{b}$ For a complete author list, see the special section of these proceedings. \\ E-mail: jwood@icecube.wisc.edu
}

The High-Altitude Water Cherenkov (HAWC) Observatory is a ground-based TeV gamma-ray observatory in the state of Puebla, Mexico at an altitude of $4100 \mathrm{~m}$ above sea level. Its $\sim 22,000$ $\mathrm{m}^{2}$ instrumented area, wide field of view ( $2 \mathrm{sr}$ ), and $>95 \%$ uptime make it an ideal instrument for discovering gamma-ray burst (GRB) emission at $>100 \mathrm{GeV}$. Such a discovery would provide key information about the origins of prompt GRB emission as well as constraints on EBL models and the violation of Lorentz invariance. We present here the results of our current GRB search methods, which include an all-sky search as well as fast follow-ups of GRBs reported by satellites, after one and a half years of data with the full HAWC detector.

35th International Cosmic Ray Conference - ICRC2017 -

10-20 July, 2017

Bexco, Busan, Korea

* Speaker. 


\section{Introduction}

The HAWC Observatory is a ground-based TeV gamma-ray observatory located in the state of Puebla, Mexico at an altitude of $4100 \mathrm{~m}$ above sea level. Its $\sim 22,000 \mathrm{~m}^{2}$ instrumented area, wide field of view ( $2 \mathrm{sr}$ ), and $>95 \%$ uptime allow it to continuously monitor for Northern hemisphere transients with emission extending up to $100 \mathrm{GeV}$ and beyond. This is particularly important for detecting gamma-ray burst (GRB) transients which have been measured up to $95 \mathrm{GeV}$ but typically have prompt emission durations shorter than the slewing times of imaging atmospheric Cherenkov telescopes.

Measurements of the highest energy gamma-rays associated with GRBs are key to developing models of the relativistic jets powering emission. This is because observations of a spectral cutoff at the highest photon energies can be interpreted as estimates of the bulk Lorentz factor $\Gamma$ in the region where gamma-rays are produced [1], providing insight into the internal GRB environment as well as the expected neutrino flux from GRBs which is sensitive to $\Gamma$ [2]. Alternatively, interpreting the spectral cutoff as attenuation of GRB photons from pair-production on extra-galactic background light (EBL) provides constraints on EBL density over cosmological distances [3].

\section{HAWC GRB Program Overview}

The HAWC GRB program presently consists of two dedicated analyses, a self-triggered all-sky search and rapid response follow-ups of GRBs reported by satellites. Both methods are performed in real-time using the quick-look air shower reconstruction produced at the HAWC site with a latency of $\sim 5$ seconds. Additionally, each search is repeated on archival data when improved calibrations and reconstruction algorithms become available.

The self-triggered all-sky method continuously searches for GRB transients at energies $>100$ $\mathrm{GeV}$ with three sliding time windows of lengths $0.2,1$, and 10 seconds which are typical of peak structures within GRB light curves. We perform the search by shifting each window forward in time by $10 \%$ its width and binning air shower events during that window using a grid of $2.1^{\circ} \times$ $2.1^{\circ}$ square spatial bins covering all points within $50^{\circ}$ of detector zenith [4]. We then compare the number of showers in each spatial bin to the expectation from charged cosmic-ray backgrounds. Locations with an air shower excess corresponding to a post-trials false alarm rate of 1 event per day are considered candidates for GRB transients and reported internally within HAWC.

The method for rapid response follow-up of reported GRBs is simpler in that it fixes the search window start time to match the external trigger time provided by a satellite. In addition, the spatial portion of the search is restricted to the reported error on the GRB location. However the basic idea is the same with air shower events during the search window binned into spatial bins and compared against the expectation from charged cosmic-ray backgrounds [5]. In cases when $T_{90}$ is available with the external trigger we search for emission occurring within $T_{90}$ as well as $3 \times T_{90}, 10 \times T_{90}$ for long GRBs and 6, 20 seconds for short GRBs with the longer windows covering possible extended emission. Otherwise we perform the follow-up with timescales of 1 and 20 seconds to cover typical $T_{90}$ values as well as a 300 second window to look for extended emission.

From the sensitivity of the HAWC Observatory we expect $\sim 0.5$ GRB detections per year from following satellite reported GRBs alone assuming most GRBs do not contain an intrinsic cutoff 
below $200 \mathrm{GeV}$ [6]. The all-sky search method yields a similar expectation as the trials penalty taken when searching the full sky is roughly compensated by the ability to search data without satellite coverage [4]. Thus far there have been no significant detections of GRB transients in the first 1.5 years of data taking since the inauguration of the full HAWC detector on March 19, 2015. This is still consistent with our expectations.

\section{Results}

Given the absence of a detection we have placed upper limits on very high energy (VHE) emission above $80 \mathrm{GeV}$ occurring within the reported $T_{90}$ of 64 GRBs detected by satellites within the HAWC field of view during the first 1.5 years with the full detector (Figure 1). This includes a limit on the third brightest burst seen by the Fermi Gamma-ray Burst Monitor, GRB 170206A, which was also detected by the Fermi Large Area Telescope (Figure 2). We derive these limits assuming an $E^{-2}$ power-law spectrum for each GRB attenuated according to EBL absorption with the fiducial model by Gilmore et al. [3] for two different redshifts, $z=0.3,1.0$. See reference [5] for more details.
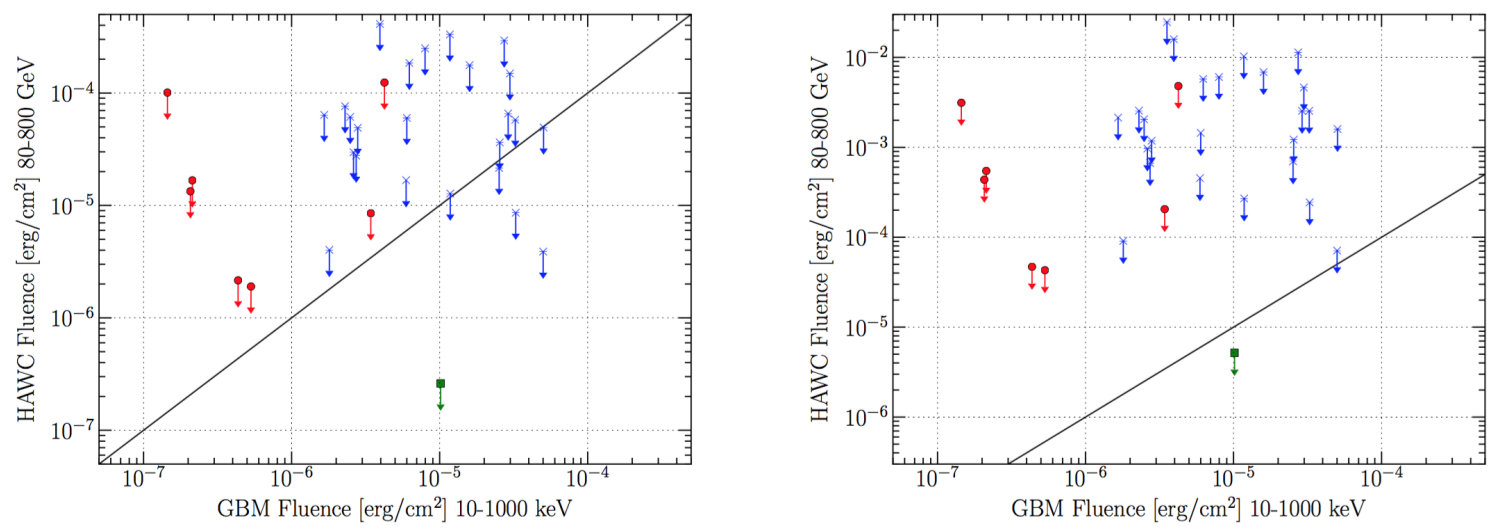

Figure 1: Comparison between the Fermi-GBM fluence and the fluence implied by the HAWC upper limits obtained during the same time period for all GRBs completely inside the HAWC field of view for the different redshifts (left $\mathrm{z}=0.3$, right $\mathrm{z}=1.0$ ). The circles (red) show short GRBs, the asterisks (blue) long GRBs and the green square GRB 170206A. The black line shows an equal fluence in the Fermi-GBM and HAWC energy range. See reference [5] for the complete list of GRBs contributing to these plots. 


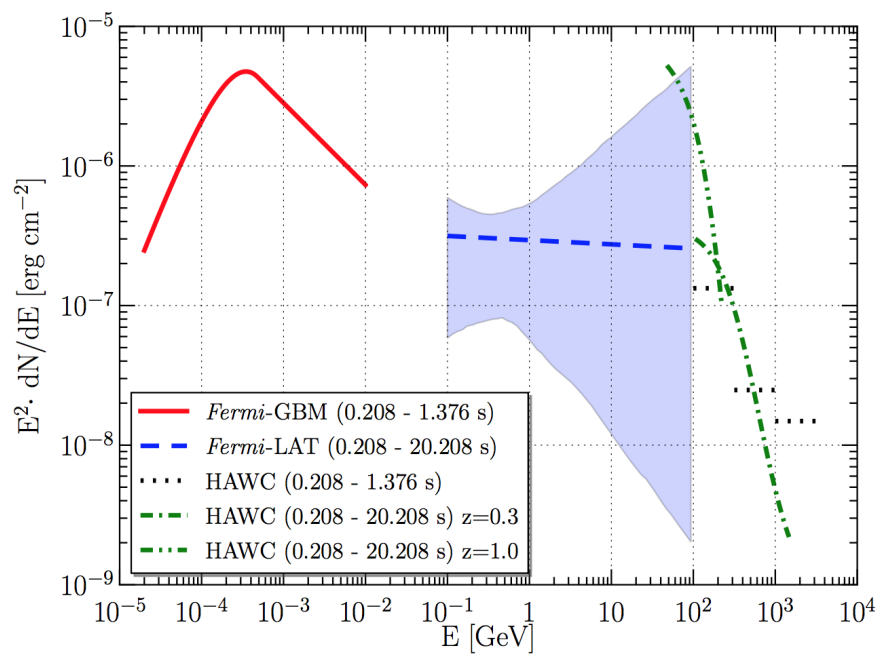

Figure 2: Solid red line shows the spectrum fitted to the prompt Fermi-GBM data of GRB 170206A, while dotted black lines show the quasi-differential HAWC limits assuming an $E^{-2}$ spectrum obtained from the HAWC data taken during the same time period. The dashed blue line shows the best fit spectrum obtained from the Fermi-LAT data in the early afterglow and the shaded area the uncertainty taking into account correlations and non-linearity of fit parameters. The green dasheddotted lines show the HAWC limits for two different assumed redshifts.

The best GRB candidate found in the all-sky search method during the first 1.5 years of operating the full HAWC detector is a 1 second transient with a pre-trial probability of $9 \times 10^{-15}$. Table 1 presents the details of this candidate and Figure 3 shows its light curve and sky map. Accounting for the effective trials taken when searching the full sky over this time period with three search window durations of lengths $0.2,1$, and 10 seconds results in a post-trial probability of 0.19 . Although this result is not significant, it does show the all-sky search algorithm can successfully find interesting event excesses within the HAWC dataset.

\begin{tabular}{|l|c|}
\hline \multicolumn{2}{|l|}{ Best All-sky GRB Search Candidate } \\
\hline Date & $2015 / 05 / 30$ \\
Trigger Time & $08: 20: 59.67$ UTC \\
Duration & 1.0 second \\
Obs. Counts & 9 \\
Bkg. Counts & 0.115 \\
Right Ascension & $292.83^{\circ}(\mathrm{J} 2000)$ \\
Declination & $-17.53^{\circ}(\mathrm{J} 2000)$ \\
Zenith & $40.48^{\circ}$ \\
\hline
\end{tabular}

Table 1: Details of the overall best candidate from the all-sky GRB search with 1.5 years of data. 

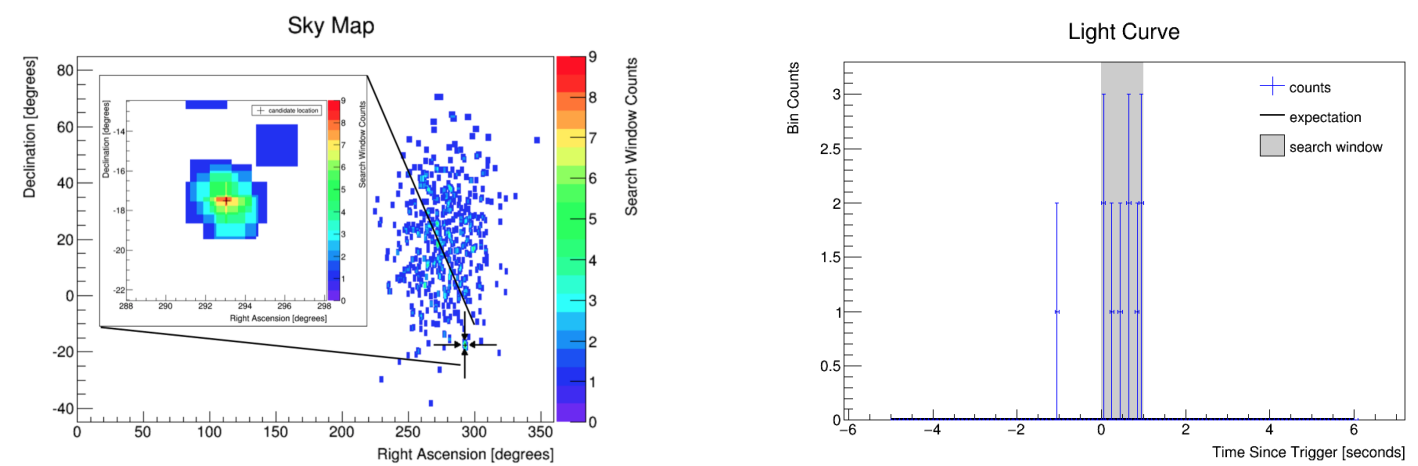

Figure 3: (Left) Sky map of detected air shower counts for the 1 second long time window containing the best candidate event from the all-sky GRB search. The cross marking the location of the candidate is at a detector zenith angle of $40.5^{\circ}$. (Right) Light curve of event counts binned in intervals of 0.1 seconds for the location of the best candidate. The 1 second window containing the best candidate is shaded in grey. The black line marks the background expectation in each light curve bin. The background expecation is so low that it cannot be distinguished from zero in this plot.

\section{Testing for Delayed VHE Emission}

We recently began expanding our follow-up program of GRBs reported by satellites to test for delayed VHE emission which is a well known feature of bursts detected by the Fermi Large Area Telescope [7]. This was initially done by repeating our standard follow-up analysis with additional time windows starting in integer multiples of $T_{90}$ after the reported trigger time [8]. Additionally, we implemented the ability to pass external triggers to our all-sky analysis method in order to perform a limited sliding time window search near the trigger time and location of known GRBs which allows us to finely optimize for the start time of VHE emission when performing follow-ups.

We tested the new follow-up capability of our all-sky search by re-analyzing several of the same GRBs studied with our existing GRB follow-up analysis. This was done by setting the sliding time window duration equal to the $T_{90}$ of each burst and reducing the temporal search range to select the single $T_{90}$ window starting at the externally reported trigger time. Table 2 presents the results which are largely identical to the standard follow-up analysis. The minor differences in the number of on-source counts and background expectation arises from the use of square spatial bins in the all-sky search and circular spatial bins in the standard follow-up analysis. 


\begin{tabular}{|c|ll|lll|}
\hline GRB & \multicolumn{2}{|l|}{ Standard follow-up analysis } & \multicolumn{3}{|c|}{ All-sky analysis with external trigger } \\
\hline $160605 \mathrm{~A}$ & $\mathrm{n}=4, \quad \mathrm{bg}=0.70, \quad \mathrm{P}=5.8 \times 10^{-3}$ & $\mathrm{n}=4, \quad \mathrm{bg}=0.59 \pm 0.02$, & $\mathrm{P}=3.1 \times 10^{-3}$ \\
$160410 \mathrm{~A}$ & $\mathrm{n}=2, \quad \mathrm{bg}=2.42, \quad \mathrm{P}=0.70$ & $\mathrm{n}=2, \quad \mathrm{bg}=2.49 \pm 0.06, \quad \mathrm{P}=0.71$ \\
$160310 \mathrm{~A}$ & $\mathrm{n}=6, \quad \mathrm{bg}=4.48, \quad \mathrm{P}=0.29$ & $\mathrm{n}=5, \quad \mathrm{bg}=4.35 \pm 0.11, \quad \mathrm{P}=0.44$ \\
$151228 \mathrm{~B}$ & $\mathrm{n}=36, \quad \mathrm{bg}=39.05, \quad \mathrm{P}=0.71$ & $\mathrm{n}=38, \quad \mathrm{bg}=38.6 \pm 0.30, \quad \mathrm{P}=0.56$ \\
$151205 \mathrm{~A}$ & $\mathrm{n}=36, \quad \mathrm{bg}=32.51, \quad \mathrm{P}=0.29$ & $\mathrm{n}=34, \quad \mathrm{bg}=30.8 \pm 0.30, \quad \mathrm{P}=0.31$ \\
\hline
\end{tabular}

Table 2: Comparison of standard follow-up analysis to triggered extension of all-sky analysis for 5 selected GRBs from [5]. $n$ represents the number of air shower events arriving within $T_{90}$ inside the spatial bin at the GRB location, $b g$ is the cosmic-ray background for this spatial bin, and $P$ is the Poisson probability for observing $n$ counts given a mean of $b g$.

\section{Summary}

We continue to support and expand upon our existing GRB program. Although there were no GRB detections within the first 1.5 years of HAWC data we have begun to place upper limits on emission $>100 \mathrm{GeV}$ for satellite detected GRBs within the HAWC field of view with the most constraining limits coming from GRB 170206A. The latest addition to our GRB program is the ability to test for delayed onset VHE emission when performing follow-ups of satellite detected GRBs.

\section{Acknowledgements}

We acknowledge the support from: the US National Science Foundation (NSF); the US Department of Energy Office of High-Energy Physics; the Laboratory Directed Research and Development (LDRD) program of Los Alamos National Laboratory; Consejo Nacional de Ciencia y Tecnología (CONACyT), México (grants 271051, 232656, 260378, 179588, 239762, 254964, 271737, 258865, 243290, 132197), Laboratorio Nacional HAWC de rayos gamma; L'OREAL Fellowship for Women in Science 2014; Red HAWC, México; DGAPA-UNAM (grants IG100317, IN111315, IN111716-3, IA102715, 109916, IA102917); VIEP-BUAP; PIFI 2012, 2013, PROFOCIE 2014, 2015; the University of Wisconsin Alumni Research Foundation; the Institute of Geophysics, Planetary Physics, and Signatures at Los Alamos National Laboratory; Polish Science Centre grant DEC-2014/13/B/ST9/945; Coordinación de la Investigación Científica de la Universidad Michoacana. Thanks to Luciano Díaz and Eduardo Murrieta for technical support. 


\section{References}

[1] T. Piran, Gamma-ray bursts and the fireball model, Phys.Rept. 314 (1999) 575-667, [astro-ph/9810256].

[2] E. Waxman and J. N. Bahcall, High-energy neutrinos from cosmological gamma-ray burst fireballs, Phys.Rev.Lett. 78 (1997) 2292-2295, [astro-ph/9701231].

[3] R. C. Gilmore, P. Madau, J. R. Primack, R. S. Somerville, and F. Haardt, GeV gamma-ray attenuation and the high-redshift UV background, Mon.Not.Roy.Astron.Soc. 399 (2009) 1694, [arXiv:0905.1144].

[4] J. Wood, An all-sky search for bursts of very high energy gamma rays with HAWC. PhD thesis, University of Maryland, College Park, 2016.

[5] R. Alfaro et al., Search for very-high-energy emission from gamma-ray bursts using the first 18 months of data from the HAWC gamma-ray observatory, ApJ (2017) [arXiv: 1705.1551 ].

[6] I. Taboada and R. C. Gilmore, Prospects for the detection of GRBs with HAWC, Nucl.Instrum.Meth. A742 (2014) 276-277, [arXiv:1306.1127].

[7] M. Ackermann et al., The first Fermi LAT gamma-ray burst catalog, Astrophys.J. 738 (2011) 138, [arXiv:1303.2908].

[8] S. Dichiara et al., Search of extended or delayed TeV emission from GRBs with HAWC, Proc. 35th ICRC (2017). 